\title{
Environment-Resistant DNA Origami Crystals Bridged by Rigid DNA Rods with Adjustable Unit Cells
}

\author{
Supporting Information
}

Ningning Ma, Lizhi Dai, Zhi Chen, Min Ji, Yong Wang, Ye Tian*

College of Engineering and Applied Sciences, State Key Laboratory of Analytical Chemistry for

Life Science, Jiangsu Key Laboratory of Artificial Functional Materials, Chemistry and Biomedicine Innovation Center, Nanjing University, Nanjing, 210023, China

*Email address: ytian@nju.edu.cn;

\section{Table of Content}

\section{Materials and Synthesis Methods}

a. Design and fabrication of octahedral DNA origami frames

b. Preparation of ssDNA-functionalized gold nanoparticles

c. Synthesis of DNA rods

d. Preparation of nanoparticles/DNA lattices

e. Preparation of nanoparticles/DNA lattices in external stimuli

II. Native Polyacrylamide Gel Electrophoresis

III. Preparation of Silica-infused Crystals

IV. Electron Microscopy

a. TEM sample preparation and imaging

b. SEM sample preparation and imaging

V. Small-angle X-ray Scattering (SAXS)

VI. Calculation of Theoretical Interparticle Distance

VII. DNA Sequences 
a. Staples of octahedral DNA origami frame (PAGE purified)

b. Sticky ends of octahedral DNA origami frame (PAGE purified)

c. DNA sequences of 4DH (PAGE purified)

d. DNA sequences of 6HB-R63 (PAGE purified)

e. DNA sequences of $6 \mathrm{HB}-\mathrm{R}_{132}$ (PAGE purified)

f. Thiolated-DNA for AuNPs function (HPLC purified)

VIII. Supplementary Figures

IX. Supplementary Tables

X. Supplementary References 


\section{Materials and Synthesis Methods}

\section{a. Design and fabrication of octahedral DNA origami frames}

Octahedral DNA origami frames (DOFs) were designed by caDNAno software (http://cadnano.org/). Each edge of the frame is a six-helix-bundle (6HB) with identical lengths around $28.56 \mathrm{~nm}$ ( 84 base pairs). Octahedral DOFs were synthesized by mixing 10 nM single-stranded DNA scaffold (M13mp18, Bayou Biolabs, LLC) and 144 short DNA staples (100 nM for each one, Shanghai Sangon Biotech Co. Ltd) in buffer solution containing $1 \mathrm{mM}$ EDTA, $40 \mathrm{mM}$ tris acetate, and $12.5 \mathrm{mM}$ magnesium acetate at room temperature. The staple DNA sequences were purchased with PAGE purification and used without further purification. Then the mixture was incubated by rapidly heated from room temperature to $90{ }^{\circ} \mathrm{C}$, followed by cooling down to $20{ }^{\circ} \mathrm{C}$ over $20 \mathrm{~h}$ to fabricate the target frames (with final concentration $\sim 10 \mathrm{nM}$ ). The synthesized DNA origami monomers were used for further assembly without purification.

\section{b. Preparation of ssDNA-functionalized gold nanoparticles}

Thiol-modified ssDNA strands were firstly mixed with TCEP (Tris (carboxyethyl) phosphine hydrochloride) to reduce the disulfide bonds, and the mixture was incubated in an ice bath for $1.5 \mathrm{~h}$. After purification, the monothiol DNA strands were mixed with $10 \mathrm{~nm}$ AuNPs (Ted Pella, Inc.) with a molar ratio of 300:1 (ssDNA:AuNPs) and incubated at room temperature for $1.5 \mathrm{~h}$. Then the mixture was buffered into $10 \mathrm{mM}$ phosphate and incubated for another $1.5 \mathrm{~h}$. After that, $\mathrm{NaCl}$ was added into the solution gradually over at least $5 \mathrm{~h}$ to reach the final concentration of $0.3 \mathrm{M}$. The prepared mixture was aged at room temperature for $18 \mathrm{~h}$. Finally, the excessive ungrafted thiolated-DNA were removed by centrifugation at $20000 \mathrm{rcf}$ for $1 \mathrm{~h}$ and washed by 0.1 M PBS buffer (0.1 M NaCl, $10 \mathrm{mM}$ phosphate) for three times.

\section{c. Synthesis of DNA rods}

Duplex DNA of $4 \mathrm{DH}$ (4DH-R $21,4 \mathrm{DH}-\mathrm{R}_{31}, 4 \mathrm{DH}-\mathrm{R}_{36}$, and $\left.4 \mathrm{DH}-\mathrm{R}_{42}\right)$ was 
synthesized by mixing equal molar ratio of two complementary ssDNA in $0.3 \mathrm{M}$ PBS $(0.3 \mathrm{M} \mathrm{NaCl}, 10 \mathrm{mM}$ phosphate) buffer, after which the mixture was cooled down from $95{ }^{\circ} \mathrm{C}$ to room temperature over two days. The formed duplex DNA was confirmed using polyacrylamide gel electrophoresis, as shown in Figure S2 and Table S1.

$6 \mathrm{HB}-\mathrm{R}_{63}$ was synthesized by mixing equal molar ratio of twenty complementary ssDNA in $12.5 \mathrm{mM}$ magnesium acetate buffer. The mixture was then cooled down from $90{ }^{\circ} \mathrm{C}$ to room temperature over two days. The formed $6 \mathrm{HB}-\mathrm{R}_{63}$ was verified by TEM, as shown in Figure S3.

$6 \mathrm{HB}-\mathrm{R}_{132}$ was synthesized by mixing equal molar ratio of $6 \mathrm{HB}-\mathrm{R}_{63}-\mathrm{I}$ and $6 \mathrm{HB}-\mathrm{R}_{63}-$ II, and cooling down from $50{ }^{\circ} \mathrm{C}$ to room temperature for around $60 \mathrm{~h}$. Representative negative stained TEM images of formed $6 \mathrm{HB}-\mathrm{R}_{132}$ was shown in Figure S12.

\section{d. Preparation of nanoparticles/DNA lattices}

$100 \mu \mathrm{L}$ octahedral DOFs prepared as mentioned above (with concentration around $10 \mathrm{nM}$ and without further purification) were mixed with excess ssDNA-functionalized AuNPs and DNA rods in $1 \times$ TAE buffer containing $12.5 \mathrm{mM}$ magnesium acetate. The mixture was then incubated from $50{ }^{\circ} \mathrm{C}$ to $20{ }^{\circ} \mathrm{C}$ at the gradient of $-0.2{ }^{\circ} \mathrm{C} / \mathrm{h}$ for two cycles (DOF-6HB-R 132 experienced three annealing cycles).

\section{e. Preparation of nanoparticles/DNA lattices in external stimuli}

Prepared nanoparticles/DNA lattices were firstly washed repeatedly with $12.5 \mathrm{mM}$ magnesium acetate buffer to remove excess DNA and nanoparticles.

For salt-stimulated systems (denoted as $S_{\text {salt }}$ ), the obtained lattices were further washed by removing the supernatant and then resuspending in $1 \times$ TAE buffer with 102.5 mM magnesium acetate.

For organic solvent-stimulated systems (containing ethanol, denoted as $S_{E t O H}$ ), $20 \%$ EtOH (volume percentages of $\mathrm{EtOH}$ in $\mathrm{H}_{2} \mathrm{O}$, containing $12.5 \mathrm{mM}$ magnesium acetate and $1 \times$ TAE buffer) was used to further washing and resuspending the obtained lattices.

For polymer-stimulated systems (containing PEG, denoted as $S_{P E G}$ ), $\sim 45 \mu \mathrm{L} 22 \%$ 
w/v PEG (M.W. $=2000$, contains $0.5 \mathrm{M} \mathrm{NaCl}, 10 \mathrm{mM}$ phosphate) was added into $\sim 5 \mu \mathrm{L}$ prepared sample. The mixed solution contains $\sim 20 \% \mathrm{w} / \mathrm{v}$ PEG, $\sim 0.45 \mathrm{M} \mathrm{NaCl}$ and $\sim 1.25 \mathrm{mM}$ magnesium acetate.

\section{Native Polyacrylamide Gel Electrophoresis}

The gel containing $12 \%$ polyacrylamide (29:1 acrylamide/bisacrylamide) was run on a JY300C electrophoresis unit at a constant voltage of $150 \mathrm{~V}$ at room temperature for $1 \mathrm{~h}$. The running buffer consists of $89 \mathrm{mM}$ Tris-boric and $2 \mathrm{mM}$ EDTA (1×TBE), and the $\mathrm{PH}$ is around 8.3. After electrophoresis, the gel was stained with $3 \mu \mathrm{L}$ SYBR Gold Nucleic Acid Gel Stain (Invitrogen) for $20 \mathrm{~min}$.

\section{Preparation of Silica-infused Crystals}

To stabilize the DNA lattices for characterization, the assembled crystal was encapsulated by silica according to the reported method. ${ }^{1-3}$ Briefly, $100 \mu \mathrm{L}$ sample solution containing DNA origami lattices prepared as mentioned above (with aggregates sitting in the bottom of the tube) was firstly washed by new buffer solution for several times. Specifically, the supernatant of the lattices sample was carefully removed and replaced by $40 \sim 50 \mu \mathrm{L} 7 \mathrm{mM} \mathrm{MgCl}_{2}(1 \times \mathrm{TAE})$ without disturbing the aggregates. The washing cycles were repeated for 7 8 times before next step. Final volume of sample solution was always fixed to $40 \mu \mathrm{L}$. Then the solution was mixed with $\quad 0.5-0.9 \mu \mathrm{L}$ TMAPS $\quad(N$-trimethoxysilylpropyl- $N, N, N$-trimethylammonium chloride) at room temperature and then shaken at $400 \mathrm{rpm}$ for $20 \mathrm{~min}$. Subsequently, 0.4-0.7 $\mu \mathrm{L}$ TEOS (tetraethyl orthosilicate) was added and the mixture was shaken for another $30 \mathrm{~min}$. The prepared solution was then left steadily for $10 \mathrm{~h}$ at room temperature. The excess silica chunks were removed by washing with ultrapure water for several times.

\section{Electron Microscopy}

\section{a. TEM sample preparation and imaging}

$5 \mu \mathrm{L}$ sample solution was deposited onto a glow-discharged carbon coated copper grid and incubated for $5 \mathrm{~min}$. The excessive solution was wicked away by filter paper. 
After washing by ultrapure water twice, the sample was negatively stained by $5 \mu \mathrm{L} 2 \%$ $(\mathrm{w} / \mathrm{v})$ uranyl acetate aqueous solution for $10 \mathrm{~s}$ and the excess staining solution was immediately wicked away. The prepared sample was observed by JEM-2100 transmission electron microscope (JEOL) and JEM-2800 transmission electron microscope (JEOL) operated at $200 \mathrm{kV}$.

\section{b. SEM sample preparation and imaging}

3-5 $\mu \mathrm{L}$ silica-infused sample was dropped onto ethanol-washed silicon slice and dried under an infrared light. The prepared samples were observed by Zeiss Ultra Plus FE-SEM (Zeiss) and JSM-7800F (JEOL) without any metal sputtering.

\section{Small-angle X-ray Scattering (SAXS)}

SAXS experiments were carried out at BL16B1/BL19U2 beamline at the Shanghai Synchrotron Radiation Facility (SSRF). The two-dimensional (2D) scattering data were collected with Pilatus $2 \mathrm{M}$ detector and integrated into 1D I $(q)$ scattering curve as a function of the scattering vector $q$ that was calculated according to $q=4 \frac{\pi}{\lambda} \sin \left(\frac{\theta}{2}\right)$, where $\lambda$ is the wavelength of incident $\mathrm{X}$-ray, $\theta$ is the scattering angle. In order to eliminate the interference of un-aggregated nanoparticles, $\mathrm{I}(q)$ was divided by the corresponding particle form factor $\mathrm{P}(q)$ and the structure factor $\mathrm{S}(q)$ was obtained. By using PowderCell software we generated the simulated data pattern, and confirmed that the lattice type of the study system was simple cubic. The unit cell parameter $d$ was measured using the following equation $d=\frac{2 \pi}{q_{0}}$, where $q_{0}$ is the scattering vector of the first peak. It should be stressed here that the same samples exhibited slight numerical differences at different beamlines. Therefore, to avoid unnecessary misunderstanding caused by different beamlines, all of the experiments here were compared at the same one.

\section{Calculation of Theoretical Interparticle Distance}

The geometric models of the octahedral DOFs and the binding details of the two octahedra nearby were shown in Figure S4. The theory distance between nanoparticle 
nearest neighbors within DOFs lattices can be determined as:

$$
d_{\text {theory }}=D+[R+2 \times l] \times 0.34+S_{S S D N A}
$$

where $d_{\text {theory }}$ is the theory interparticle distance calculated from the geometric models, $D$ describing the half wide of the octahedra and set to be $22.18 \mathrm{~nm}$ taking the chamfering of the vertex into account, $R$ is the length of DNA rods, $l$ is the recognition parts with 8 bases. $S_{S S D N A}$ is the averaged end-to-end distance of single-stranded DNA, which can be applied with an ideal chain model $^{4,5}$ and expressed as:

$$
S_{S S D N A}=2(\sqrt{\mathrm{m}}+\sqrt{\mathrm{n}}) \times 0.6
$$

where $m$ is the number of free bases of the sticky ends designed in the DNA origami part, while $n$ is the number of free bases of the sticky ends designed in the DNA rods part (Figure S4). The relevant values and calculation results were listed in Table S2.

\section{DNA Sequences}

\section{a. Staples of octahedral DNA origami frame (PAGE purified)}

octa -1 TCAAAGCGAACCAGACCGTTTTATATAGTC

octa-2 GCTTTGAGGACTAAAGAGCAACGGGGAGTT

octa-3 GTAAATCGTCGCTATTGAATAACTCAAGAA

octa-4 AAGCCTTAAATCAAGACTTGCGGAGCAAAT

octa -5 ATTTTAAGAACTGGCTTGAATTATCAGTGA

octa-6 GTTAAAATTCGCATTATAAACGTAAACTAG

octa -7 AGCACCATTACCATTACAGCAAATGACGGA

octa-8 ATTGCGTAGATTTTCAAAACAGATTGTTTG

octa-9 TAACCTGTTTAGCTATTTTCGCATTCATTC

octa-10 GTCAGAGGGTAATTGAGAACACCAAAATAG

octa-11 CTCCAGCCAGCTTTCCCCTCAGGACGTTGG

octa-12 GTCCACTATTAAAGAACCAGTTTTGGTTCC

octa-13 TAAAGGTGGCAACATAGTAGAAAATAATAA

octa-14 GATAAGTCCTGAACAACTGTTTAAAGAGAA

octa-15 GGTAATAGTAAAATGTAAGTTTTACACTAT

octa-16 TCAGAACCGCCACCCTCTCAGAGTATTAGC

octa-17 AAGGGAACCGAACTGAGCAGACGGTATCAT

octa-18 GTAAAGATTCAAAAGGCCTGAGTTGACCCT

octa-19 AGGCGTTAAATAAGAAGACCGTGTCGCAAG

octa-20 CAGGTCGACTCTAGAGCAAGCTTCAAGGCG

octa-21 CAGAGCCACCACCCTCTCAGAACTCGAGAG

octa-22 TTCACGTTGAAAATCTTGCGAATGGGATTT

octa-23 AAGTTTTAACGGGGTCGGAGTGTAGAATGG

octa-24 TTGCGTATTGGGCGCCCGCGGGGTGCGCTC 
octa-25 GTCACCAGAGCCATGGTGAATTATCACCAATCAGAAAAGCCT

octa-26 GGACAGAGTTACTTTGTCGAAATCCGCGTGTATCACCGTACG

octa-27 CAACATGATTTACGAGCATGGAATAAGTAAGACGACAATAAA

octa-28 AACCAGACGCTACGTTAATAAAACGAACATACCACATTCAGG

octa-29 TGACCTACTAGAAAAAGCCCCAGGCAAAGCAATTTCATCTTC

octa-30 TGCCGGAAGGGGACTCGTAACCGTGCATTATATTTTAGTTCT

octa-31 AGAACCCCAAATCACCATCTGCGGAATCGAATAAAAATTTTT

octa-32 GCTCCATTGTGTACCGTAACACTGAGTTAGTTAGCGTAACCT

octa-33 AGTACCGAATAGGAACCCAAACGGTGTAACCTCAGGAGGTTT

octa-34 CAGTTTGAATGTTTAGTATCATATGCGTAGAATCGCCATAGC

octa-35 AAGATTGTTTTTTAACCAAGAAACCATCGACCCAAAAACAGG

octa-36 TCAGAGCGCCACCACATAATCAAAATCAGAACGAGTAGTATG

octa-37 GATGGTTGGGAAGAAAAATCCACCAGAAATAATTGGGCTTGA

octa-38 CTCCTTAACGTAGAAACCAATCAATAATTCATCGAGAACAGA

octa-39 AGACACCTTACGCAGAACTGGCATGATTTTCTGTCCAGACAA

octa-40 GCCAGCTAGGCGATAGCTTAGATTAAGACCTTTTTAACCTGT

octa -41 CCGACTTATTAGGAACGCCATCAAAAATGAGTAACAACCCCA

octa-42 GTCCAATAGCGAGAACCAGACGACGATATTCAACGCAAGGGA

octa-43 CCAAAATACAATATGATATTCAACCGTTAGGCTATCAGGTAA

octa-44 AACAGTACTTGAAAACATATGAGACGGGTCTTTTTTAATGGA

octa-45 TTTCACCGCATTAAAGTCGGGAAACCTGATTTGAATTACCCA

octa-46 GAGAATAGAGCCTTACCGTCTATCAAATGGAGCGGAATTAGA

octa -47 ATAATTAAATTTAAAAAACTTTTTCAAACTTTTAACAACGCC

octa-48 GCACCCAGCGTTTTTTATCCGGTATTCTAGGCGAATTATTCA

octa-49 GGAAGCGCCCACAAACAGTTAATGCCCCGACTCCTCAAGATA

octa-50 GTTTGCCTATTCACAGGCAGGTCAGACGCCACCACACCACCC

octa-51 CGCGAGCTTAGTTTTTCCCAATTCTGCGCAAGTGTAAAGCCT

octa-52 AGAAGCAACCAAGCCAAAAGAATACACTAATGCCAAAACTCC

octa-53 ATTAAGTATAAAGCGGCAAGGCAAAGAAACTAATAGGGTACC

octa-54 CAGTGCCTACATGGGAATTTACCGTTCCACAAGTAAGCAGAT

octa-55 ATAAGGCGCCAAAAGTTGAGATTTAGGATAACGGACCAGTCA

octa-56 TGCTAAACAGATGAAGAAACCACCAGAATTTAAAAAAAGGCT

octa-57 CAGCCTTGGTTTTGTATTAAGAGGCTGACTGCCTATATCAGA

octa-58 CGGAATAATTCAACCCAGCGCCAAAGACTTATTTTAACGCAA

octa-59 CGCCTGAATTACCCTAATCTTGACAAGACAGACCATGAAAGA

octa-60 ACGCGAGGCTACAACAGTACCTTTTACAAATCGCGCAGAGAA

octa-61 CAGCGAACATTAAAAGAGAGTACCTTTACTGAATATAATGAA

octa-62 GGACGTTTAAATTTCGACGAGAAACACCACCACTAATGCAGAT

octa-63 AAAGCGCCAAAGTTTATCTTACCGAAGCCCAATAATGAGTAA

octa-64 GAGCTCGTTGTAAACGCCAGGGTTTTCCAAAGCAATAAAGCC

octa-65 AATTATTGTTTTCATGCCTTTAGCGTCAGATAGCACGGAAAC

octa-66 AAGTTTCAGACAGCCGGGATCGTCACCCTTCTGTAGCTCAAC

octa-67 ACAAAGAAATTTAGGTAGGGCTTAATTGTATACAACGGAATC

octa-68 AACAAAAATAACTAGGTCTGAGAGACTACGCTGAGTTTCCCT 
octa-69 CATAACCTAAATCAACAGTTCAGAAAACGTCATAAGGATAGC

octa-70 CACGACGAATTCGTGTGGCATCAATTCTTTAGCAAAATTACG

octa-71 CCTACCAACAGTAATTTTATCCTGAATCAAACAGCCATATGA

octa-72 GATTATAAAGAAACGCCAGTTACAAAATTTACCAACGTCAGA

octa-73 AGTAGATTGAAAAGAATCATGGTCATAGCCGGAAGCATAAGT

octa-74 TAGAATCCATAAATCATTTAACAATTTCTCCCGGCTTAGGTT

octa-75 AAAGGCCAAATATGTTAGAGCTTAATTGATTGCTCCATGAGG

octa-76 CCAAAAGGAAAGGACAACAGTTTCAGCGAATCATCATATTCC

octa-77 GAAATCGATAACCGGATACCGATAGTTGTATCAGCTCCAACG

octa-78 TGAATATTATCAAAATAATGGAAGGGTTAATATTTATCCCAA

octa-79 GAGGAAGCAGGATTCGGGTAAAATACGTAAAACACCCCCCAG

octa-80 GGTTGATTTTCCAGCAGACAGCCCTCATTCGTCACGGGATAG

octa-81 CAAGCCCCCACCCTTAGCCCGGAATAGGACGATCTAAAGTTT

octa-82 TGTAGATATTACGCGGCGATCGGTGCGGGCGCCATCTTCTGG

octa-83 CATCCTATTCAGCTAAAAGGTAAAGTAAAAAGCAAGCCGTTT

octa-84 CAGCTCATATAAGCGTACCCCGGTTGATGTGTCGGATTCTCC

octa-85 CATGTCACAAACGGCATTAAATGTGAGCAATTCGCGTTAAAT

octa-86 AGCGTCACGTATAAGAATTGAGTTAAGCCCTTTTTAAAGAAAG

octa-87 TATAAAGCATCGTAACCAAGTACCGCACCGGCTGTAATATCC

octa-88 ATAGCCCGCGAAAATAATTGTATCGGTTCGCCGACAATGAGT

octa-89 AGACAGTTCATATAGGAGAAGCCTTTATAACATTGCCTGAGA

octa-90 AACAGGTCCCGAAATTGCATCAAAAAGATCTTTGATCATCAG

octa-91 ACTGCCCTTGCCCCGTTGCAGCAAGCGGCAACAGCTTTTTCT

octa-92 TCAAAGGGAGATAGCCCTTATAAATCAAGACAACAACCATCG

octa-93 GTAATACGCAAACATGAGAGATCTACAACTAGCTGAGGCCGG

octa-94 GAGATAACATTAGAAGAATAACATAAAAAGGAAGGATTAGGA

octa-95 CAGATATTACCTGAATACCAAGTTACAATCGGGAGCTATTTT

octa-96 CATATAACTAATGAACACAACATACGAGCTGTTTCTTTTGGG

octa-97 ATGTTTTGCTTTTGATCGGAACGAGGGTACTTTTTCTTTTGATAAGAGGTCATT

octa-98 CTTCGCTGGGCGCAGACGACAGTATCGGGGCACCGTCGCCATTCAGGCTGCGCA

octa-99 GATATTCTAAATTGAGCCGGAACGAGGCCCAACTTGGCGCATAGGCTGGCTGAC

octa-100 TGTCGTCATAAGTACAGAACCGCCACCCATTTTCACAGTACAAACTACAACGCC

octa-101 CGATTATAAGCGGAGACTTCAAATATCGCGGAAGCCTACGAAGGCACCAACCTA

octa-102 AACATGTACGCGAGTGGTTTGAAATACCTAAACACATTCTTACCAGTATAAAGC

octa-103 GTCTGGATTTTGCGTTTTAAATGCAATGGTGAGAAATAAATTAATGCCGGAGAG octa-104 GCCTTGAATCTTTTCCGGAACCGCCTCCCAGAGCCCAGAGCCGCCGCCAGCATT octa-105 CGCTGGTGCTTTCCTGAATCGGCCAACGAGGGTGGTGATTGCCCTTCACCGCCT octa-106 ACATAACTTGCCCTAACTTTAATCATTGCATTATAACAACATTATTACAGGTAG octa-107 TTATTTTTACCGACAATGCAGAACGCGCGAAAAATCTTTCCTTATCATTCCAAG octa-108 TTTCAATAGAAGGCAGCGAACCTCCCGATTAGTTGAAACAATAACGGATTCGCC octa-109 GGGCGACCCCAAAAGTATGTTAGCAAACTAAAAGAGTCACAATCAATAGAAAAT octa-110 ATGACCACTCGTTTGGCTTTTGCAAAAGTTAGACTATATTCATTGAATCCCCCT octa-111 TCCAAATCTTCTGAATTATTTGCACGTAGGTTTAACGCTAACGAGCGTCTTTCC octa-112 GGGTTATTTAATTACAATATATGTGAGTAATTAATAAGAGTCAATAGTGAATTT 
octa-113 ATCCATCACTTCATACTCTACGTTGTTGTTGTTGTTGTTGGGGTGCCAGTTGAGACCATTAGATACAATTTTCACTGTGTGAAATTGTTATCC oc ta-114 ATCCATCACTTCATACTCTACGTTGTTGTTGTTGTTGTTTCAGAGCTGGGTAAACGACGGCCAGTGCGATCCCCGTAGTAGCATTAACATCCA octa-115 ATCCATCACTTCATACTCTACGTTGTTGTTGTTGTTGTTTTAGGGGTACAGAGCGGGAGAATTAACTGCGCTAATTTCGGAACCTATTATTCT octa-116 ATCCATCACTTCATACTCTACGTTGTTGTTGTTGTTGTTTGATTATCAACTTTACAACTAAAGGAATCCAAAAAGTTTGAGTAACATTATCAT oc ta-117 ATCCATCACTTCATACTCTACGTTGTTGTTGTTGTTGTTGTAGCGCCATTAAATTGGGAATTAGAGCGCAAGGCGCACCGTAATCAGTAGCGA oc ta-118 ATCCATCACTTCATACTCTACGTTGTTGTTGTTGTTGTTAGCGGAAAGTCTCTCTTTTGATGATACAAGTGCCTTAAGAGCAAGAAACAATGA octa-119 ATCCATCACTTCATACTCTACGTTGTTGTTGTTGTTGTTGTGGGAAATCATATAAATATTTAAATTGAATTTTTGTCTGGCCTTCCTGTAGCC oc ta-120 ATCCATCACTTCATACTCTACGTTGTTGTTGTTGTTGTTCCCACGCGCAAAATGGTTGAGTGTTGTTCGTGGACTTGCTTTCGAGGTGAATTT Note: staples from 113 to 120 are inner DNA designed for AuNPs catching and ULTRAPAGE purified.

\section{b. Sticky ends of octahedral DNA origami frame (PAGE purified)}

\section{i) $\boldsymbol{m}=\mathbf{0}$}

1 CAAATGCTTTAAAAAATCAGGTCTTTAAGAGCAGCCAGAGGGACTTGACT

2 AAAGATTCATCAGGAATTACGAGGCATGCTCATCCTTATGCGACTTGACT

3 CTTCATCAAGAGAAATCAACGTAACAGAGATTTGTCAATCATACTTGACT

4 AAACGAAAGAGGGCGAAACAAAGTACTGACTATATTCGAGCTACTTGACT

5 GGTAGCTATTTTAGAGAATCGATGAAAACATTAAATGTGTAGACTTGACT

6 ATAAATCATACATAAATCGGTTGTACTGTGCTGGCATGCCTGACTTGACT

7 ACTGTTGGGAAGCAGCTGGCGAAAGGATAGGTCAAGATCGCAACTTGACT

8 AGCTTTCATCAACGGATTGACCGTAAAATCGTATAATATTTTACTTGACT

9 GACAGGAGGTTGAAACAAATAAATCCGCCCCCTCCGCCACCCACTTGACT

10 CAGAATCAAGTTTCGGCATTTTCGGTTAAATATATCACCAGTACTTGACT

11 TCATATGGTTTACGATTGAGGGAGGGAAACGCAATACATACAACTTGACT

12 AATAGCAATAGCACCAGAAGGAAACCTAAAGCCACTGGTAATACTTGACT

13 TGTAGCATTCCAACGTTAGTAAATGAAGTGCCGCGCCACCCTACTTGACT

14 GAAACATGAAAGCTCAGTACCAGGCGAAAAATGCTGAACAAAACTTGACT

15 AGAGCCTAATTTGATTTTTTGTTTAAATCCTGAAATAAAGAAACTTGACT

16 TTTGCGGAACAATGGCAATTCATCAATCTGTATAATAATTTTACTTGACT

17 TTTGCGGATGGCCAACTAAAGTACGGGCTTGCAGCTACAGAGACTTGACT

18 CTTAAACAGCTTATATATTCGGTCGCTTGATGGGGAACAAGAACTTGACT

19 GGCCCTGAGAGAAGCAGGCGAAAATCATTGCGTAGAGGCGGTACTTGACT

20 GCTCACAATTCCGTGAGCTAACTCACTGGAAGTAATGGTCAAACTTGACT

21 CAACGCTCAACAGCAGAGGCATTTTCAATCCAATGATAAATAACTTGACT

22 ATCAAAATCATATATGTAAATGCTGAACAAACACTTGCTTCTACTTGACT

23 TGATTGCTTTGAGCAAAAGAAGATGAAATAGCAGAGGTTTTGACTTGACT

24 AACGGGTATTAAGGAATCATTACCGCCAGTAATTCAACAATAACTTGACT

\section{ii) $\boldsymbol{m}=1$}

1 CAAATGCTTTAAAAAATCAGGTCTTTAAGAGCAGCCAGAGGGTACTTGACT

2 AAAGATTCATCAGGAATTACGAGGCATGCTCATCCTTATGCGTACTTGACT

3 CTTCATCAAGAGAAATCAACGTAACAGAGATTTGTCAATCATTACTTGACT

4 AAACGAAAGAGGGCGAAACAAAGTACTGACTATATTCGAGCTTACTTGACT

5 GGTAGCTATTTTAGAGAATCGATGAAAACATTAAATGTGTAGTACTTGACT 
6 ATAAATCATACATAAATCGGTTGTACTGTGCTGGCATGCCTGTACTTGACT

7 ACTGTTGGGAAGCAGCTGGCGAAAGGATAGGTCAAGATCGCATACTTGACT

8 AGCTTTCATCAACGGATTGACCGTAAAATCGTATAATATTTTTACTTGACT

9 GACAGGAGGTTGAAACAAATAAATCCGCCCCCTCCGCCACCCTACTTGACT

10 CAGAATCAAGTTTCGGCATTTTCGGTTAAATATATCACCAGTTACTTGACT

11 TCATATGGTTTACGATTGAGGGAGGGAAACGCAATACATACATACTTGACT

12 AATAGCAATAGCACCAGAAGGAAACCTAAAGCCACTGGTAATTACTTGACT

13 TGTAGCATTCCAACGTTAGTAAATGAAGTGCCGCGCCACCCTTACTTGACT

14 GAAACATGAAAGCTCAGTACCAGGCGAAAAATGCTGAACAAATACTTGACT

15 AGAGCCTAATTTGATTTTTTGTTTAAATCCTGAAATAAAGAATACTTGACT

16 TTTGCGGAACAATGGCAATTCATCAATCTGTATAATAATTTTTACTTGACT

17 TTTGCGGATGGCCAACTAAAGTACGGGCTTGCAGCTACAGAGTACTTGACT

18 CTTAAACAGCTTATATATTCGGTCGCTTGATGGGGAACAAGATACTTGACT

19 GGCCCTGAGAGAAGCAGGCGAAAATCATTGCGTAGAGGCGGTTACTTGACT

20 GCTCACAATTCCGTGAGCTAACTCACTGGAAGTAATGGTCAATACTTGACT

21 CAACGCTCAACAGCAGAGGCATTTTCAATCCAATGATAAATATACTTGACT

22 ATCAAAATCATATATGTAAATGCTGAACAAACACTTGCTTCTTACTTGACT

23 TGATTGCTTTGAGCAAAAGAAGATGAAATAGCAGAGGTTTTGTACTTGACT

24 AACGGGTATTAAGGAATCATTACCGCCAGTAATTCAACAATATACTTGACT

\section{iii) $m=3$}

1 CAAATGCTTTAAAAAATCAGGTCTTTAAGAGCAGCCAGAGGGTTTACTTGACT

2 AAAGATTCATCAGGAATTACGAGGCATGCTCATCCTTATGCGTTTACTTGACT

3 CTTCATCAAGAGAAATCAACGTAACAGAGATTTGTCAATCATTTTACTTGACT

4 AAACGAAAGAGGGCGAAACAAAGTACTGACTATATTCGAGCTTTTACTTGACT

5 GGTAGCTATTTTAGAGAATCGATGAAAACATTAAATGTGTAGTTTACTTGACT

6 ATAAATCATACATAAATCGGTTGTACTGTGCTGGCATGCCTGTTTACTTGACT

7 ACTGTTGGGAAGCAGCTGGCGAAAGGATAGGTCAAGATCGCATTTACTTGACT

8 AGCTTTCATCAACGGATTGACCGTAAAATCGTATAATATTTTTTTACTTGACT

9 GACAGGAGGTTGAAACAAATAAATCCGCCCCCTCCGCCACCCTTTACTTGACT

10 CAGAATCAAGTTTCGGCATTTTCGGTTAAATATATCACCAGTTTTACTTGACT

11 TCATATGGTTTACGATTGAGGGAGGGAAACGCAATACATACATTTACTTGACT

12 AATAGCAATAGCACCAGAAGGAAACCTAAAGCCACTGGTAATTTTACTTGACT

13 TGTAGCATTCCAACGTTAGTAAATGAAGTGCCGCGCCACCCTTTTACTTGACT

14 GAAACATGAAAGCTCAGTACCAGGCGAAAAATGCTGAACAAATTTACTTGACT

15 AGAGCCTAATTTGATTTTTTGTTTAAATCCTGAAATAAAGAATTTACTTGACT

16 TTTGCGGAACAATGGCAATTCATCAATCTGTATAATAATTTTTTTACTTGACT

17 TTTGCGGATGGCCAACTAAAGTACGGGCTTGCAGCTACAGAGTTTACTTGACT

18 CTTAAACAGCTTATATATTCGGTCGCTTGATGGGGAACAAGATTTACTTGACT

19 GGCCCTGAGAGAAGCAGGCGAAAATCATTGCGTAGAGGCGGTTTTACTTGACT

20 GCTCACAATTCCGTGAGCTAACTCACTGGAAGTAATGGTCAATTTACTTGACT

21 CAACGCTCAACAGCAGAGGCATTTTCAATCCAATGATAAATATTTACTTGACT

22 ATCAAAATCATATATGTAAATGCTGAACAAACACTTGCTTCTTTTACTTGACT

23 TGATTGCTTTGAGCAAAAGAaGaTGAAATAGCAGAGGTTTTGTTTACTTGACT 


\title{
c. DNA sequences of 4DH (PAGE purified) \\ c1. 4DH-R21 \\ A CGACCGATGAATAGCGGTCAGAGTCAAGT \\ B CTGACCGCTATTCATCGGTCGAGTCAAGT
}

\begin{abstract}
c2. 4DH-R31
A CGACCGATGAATAGCGGTCAGATCCGTACCTAGTCAAGT

B AGGTACGGATCTGACCGCTATTCATCGGTCGAGTCAAGT
\end{abstract}

\section{c3. 4DH-R36}
A CGACCGATGAATAGCGGTCAGATCCGTACCTACTCGAGTCAAGT
B CGAGTAGGTACGGATCTGACCGCTATTCATCGGTCGAGTCAAGT

\section{c4. 4DH-R 42}

A GTATTCCATAGGTCGCCTCGCTTTGCAGATAAGCTAATATCAGTCAAGT

B GATATTAGCTTATCTGCAAAGCGAGGCGACCTATGGAATACAGTCAAGT

\section{d. DNA sequences of 6HB-R63 (PAGE purified)}

\section{AGGCTGACTGCCATTGTACTT}

2 TGCCTGCATCGTGAGTAGCTT

3 TCCTGTCTTCGTCGTGCGTGA

4 GCGGTTGAACGATCGCACGAG

5 ACTTATGAACAGGACTATCAA

6 CTACGAGTTAATAGAGCGACC

7 CTAGATCCTCCAAGTCAGGAGCTCGAAA

8 AGCTTAATTACGACAACCGCCCGTTTACAAGCACT

9 AACACTTATCAATGAGTGTGCTGCTAATGTATGTC

10 CACTCTCGGTGGACGTTCCAGGCCATACGCTGTTA

11 ACCAGCCAGGCGGATAGAGGAACATAGCTAGTCAAGT

12 GAGATGCCAGAGAATTGCTGACCGCTATTAGTCAAGT

13 CAGTACCGTGTCTTGCTACGACTTCGACTAGTCAAGT

14 AATTCGTATTCTAGTTCCGCTCTCCGAGTAACCGATAGTCAAGT

15 TAACAGCGTATGGCCTGGAACCGACGAACATAAGTAAGCTACCTAGAATTCCGCCTGGCTGGT

16 TTTCGAGCTCCTGAATTAGCATCAGCCTGGTCGCTGATCGTTGGCGGTTGTCGTAATTAAGCT

17 TCGGTTACTCGGAGAGCGGAATCACGATCTCGTAGTCACGCAGTCCACCTTCTCTGGCATCTCTAGTCAAGT

18 ATAGCGGTCAGCAAGAGAGTGCTCGTGCCTATTAAGCAGGCAGACATACCTTGGAGGATCTAGTAGTCAAGT

19 GTCGAAGTCGTAGCGTAAACGCAACCGCTTGATAGATGGCAGGCACACTCATTGATAAGTGTTTAGTCAAGT

20 GCTATGTTCCTCTAACGAATTAAGTACATCCTGTTGACAGGAAGTGCTTAAGACACGGTACTGTAGTCAAGT

\section{e. DNA sequences of 6HB-R132 (PAGE purified)} 6HB-R132-I

6HB-I-1 TAACAGCGTATGGCCTGGAACCGACGAACATAAGTAAGCTACCTAGAATTCCGCCTGGCTGGT 
6HB-I-2 AGCTTAATTACGACAACCGCCCGTTTACAAGCACT

6HB-I-3 GAAGAGAACACTTATCAATGAGTGTGCTGCTAATGTATGTC

6HB-I-4 GAAGAGACCAGCCAGGCGGATAGAGGAACATAGC

6HB-I-5 GAGATGCCAGAGAATTGCTGACCGCTATTAGTCAAGT

6HB-I-6 GAAGAGTCGGTTACTCGGAGAGCGGAATCACGATCTCGTAGTCACGCAGTCCACCTTCTCTGGCATCTCTAGTCAAGT

6HB-I-7 ATAGCGGTCAGCAAGAGAGTGCTCGTGCCTATTAAGCAGGCAGACATACCTTGGAGGATCTAG

6HB-I-8 GAAGAGCTAGATCCTCCAAGTCAGGAGCTCGAAA

6HB-I-9 GAAGAGTTTCGAGCTCCTGAATTAGCATCAGCCTGGTCGCTGATCGTTGGCGGTTGTCGTAATTAAGCT

6HB-I-10 GTCGAAGTCGTAGCGTAAACGCAACCGCTTGATAGATGGCAGGCACACTCATTGATAAGTGTT

6HB-I-11 CAGTACCGTGTCTTGCTACGACTTCGACTAGTCAAGT

6HB-I-12 GAAGAGGCTATGTTCCTCTAACGAATTAAGTACATCCTGTTGACAGGAAGTGCTTAAGACACGGTACTGTAGTCAAGT

6HB-I-13 ACTTATGAACAGGACTATCAA

6HB-I-14 CTACGAGTTAATAGAGCGACC

6HB-I-15 CACTCTCGGTGGACGTTCCAGGCCATACGCTGTTA

6HB-I-16 AGGCTGACTGCCATTGTACTT

6HB-I-17 TGCCTGCATCGTGAGTAGCTT

6HB-I-18 TCCTGTCTTCGTCGTGCGTGA

6HB-I-19 GCGGTTGAACGATCGCACGAG

6HB-I-20 AATTCGTATTCTAGTTCCGCTCTCCGAGTAACCGA

\section{HB-R132-II}

6HB-II-1 CTCTTCTAACAGCGTATGGCCTGGAACCGACGAACATAAGTAAGCTACCTAGAATTCCGCCTGGCTGGT

6HB-II-2 CTCTTCAGCTTAATTACGACAACCGCCCGTTTACAAGCACT

6HB-II-3 AACACTTATCAATGAGTGTGCTGCTAATGTATGTC

6HB-II-4 ACCAGCCAGGCGGATAGAGGAACATAGCTAGTCAAGT

6HB-II-5 CTCTTCGAGATGCCAGAGAATTGCTGACCGCTAT

6HB-II-6 TCGGTTACTCGGAGAGCGGAATCACGATCTCGTAGTCACGCAGTCCACCTTCTCTGGCATCTC

6HB-II-7 CTCTTCATAGCGGTCAGCAAGAGAGTGCTCGTGCCTATTAAGCAGGCAGACATACCTTGGAGGATCTAGTAGTCAAGT

6HB-II-8 CTAGATCCTCCAAGTCAGGAGCTCGAAA

6HB-II-9 TTTCGAGCTCCTGAATTAGCATCAGCCTGGTCGCTGATCGTTGGCGGTTGTCGTAATTAAGCT

6HB-II-10 CTCTTCGTCGAAGTCGTAGCGTAAACGCAACCGCTTGATAGATGGCAGGCACACTCATTGATAAGTGTTTAGTCAAGT

6HB-II-11 CTCTTCCAGTACCGTGTCTTGCTACGACTTCGAC

6HB-II-12 GCTATGTTCCTCTAACGAATTAAGTACATCCTGTTGACAGGAAGTGCTTAAGACACGGTACTG

6HB-II-13 ACTTATGAACAGGACTATCAA

6HB-II-14 CTACGAGTTAATAGAGCGACC

6HB-II-15 CACTCTCGGTGGACGTTCCAGGCCATACGCTGTTA

6HB-II-16 AGGCTGACTGCCATTGTACTT

6HB-II-17 TGCCTGCATCGTGAGTAGCTT

6HB-II-18 TCCTGTCTTCGTCGTGCGTGA

6HB-II-19 GCGGTTGAACGATCGCACGAG

6HB-II-20 AATTCGTATTCTAGTTCCGCTCTCCGAGTAACCGATAGTCAAGT

\section{f. Thiolated-DNA for AuNPs function (HPLC purified)} 5'-GAAGTGATGGATGAT-SH-3' 


\section{Supplementary Figures}

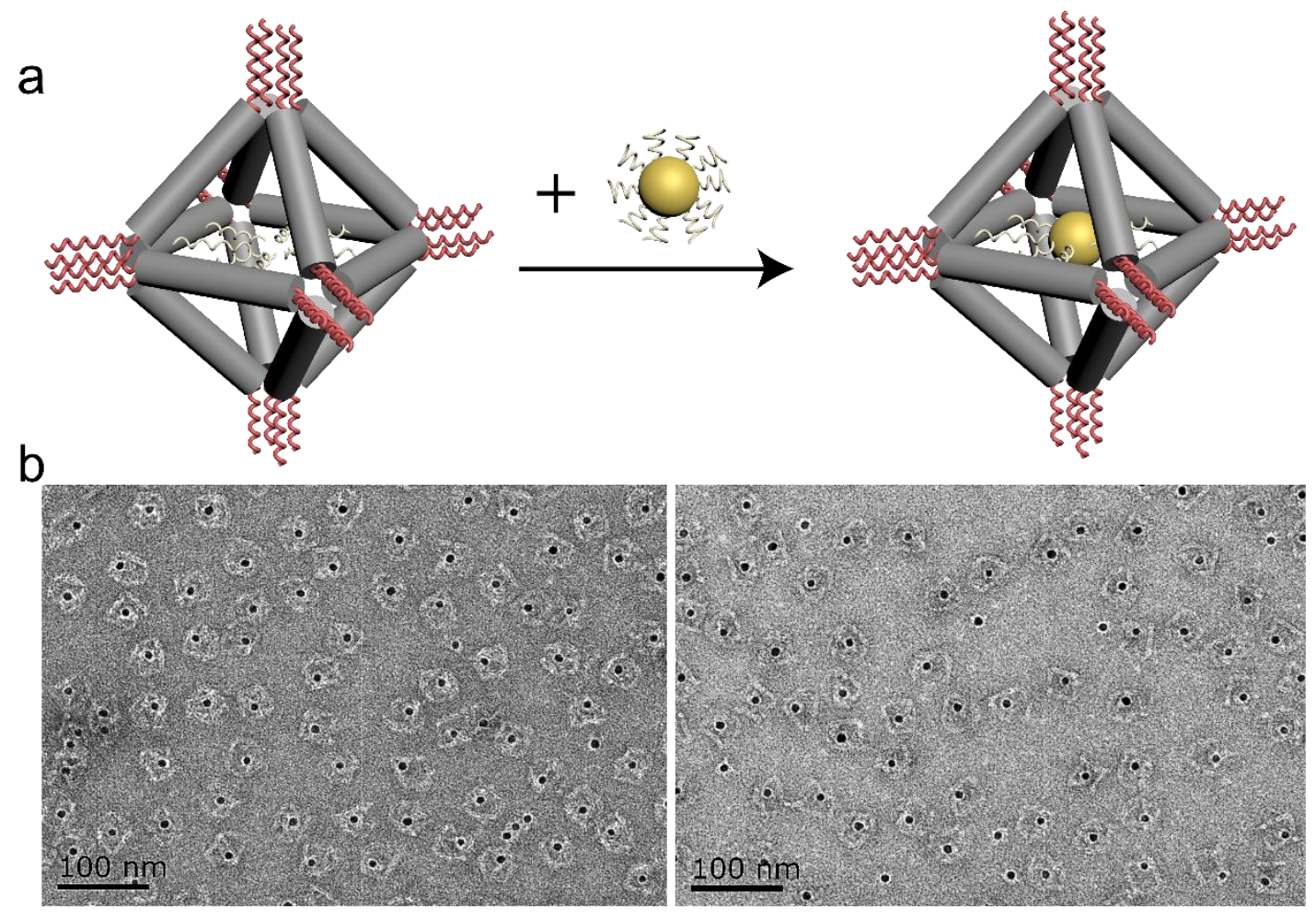

Figure S1. (a) Schematic illustration for inserting gold nanoparticles into octahedral

DOFs. (b) Representative negative-stained TEM images of the octahedral DOFs with $10 \mathrm{~nm}$ AuNPs inserted. 
a
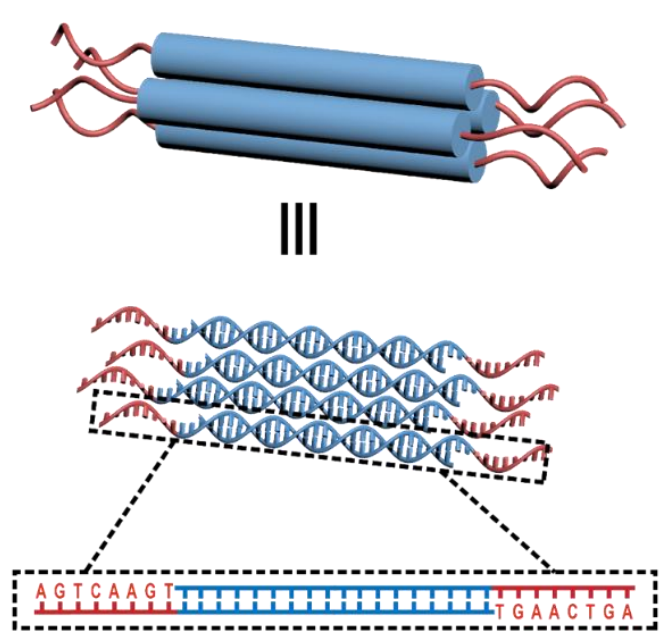

b

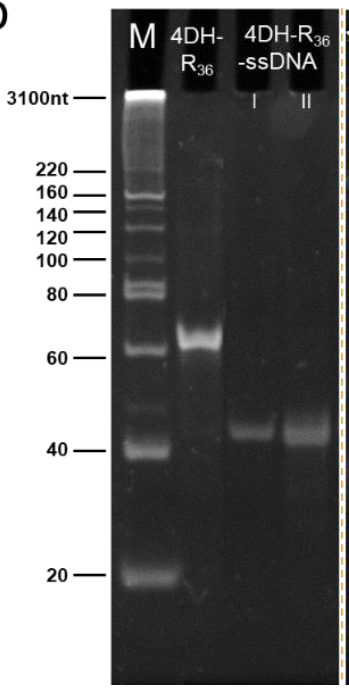

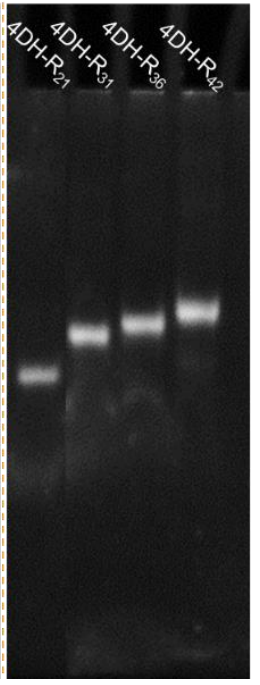

Figure S2. (a) Structure diagram of 4DH. (b) $12 \%$ PAGE gel analysis of duplex DNA of 4DH. M corresponded to the DNA ladder. 

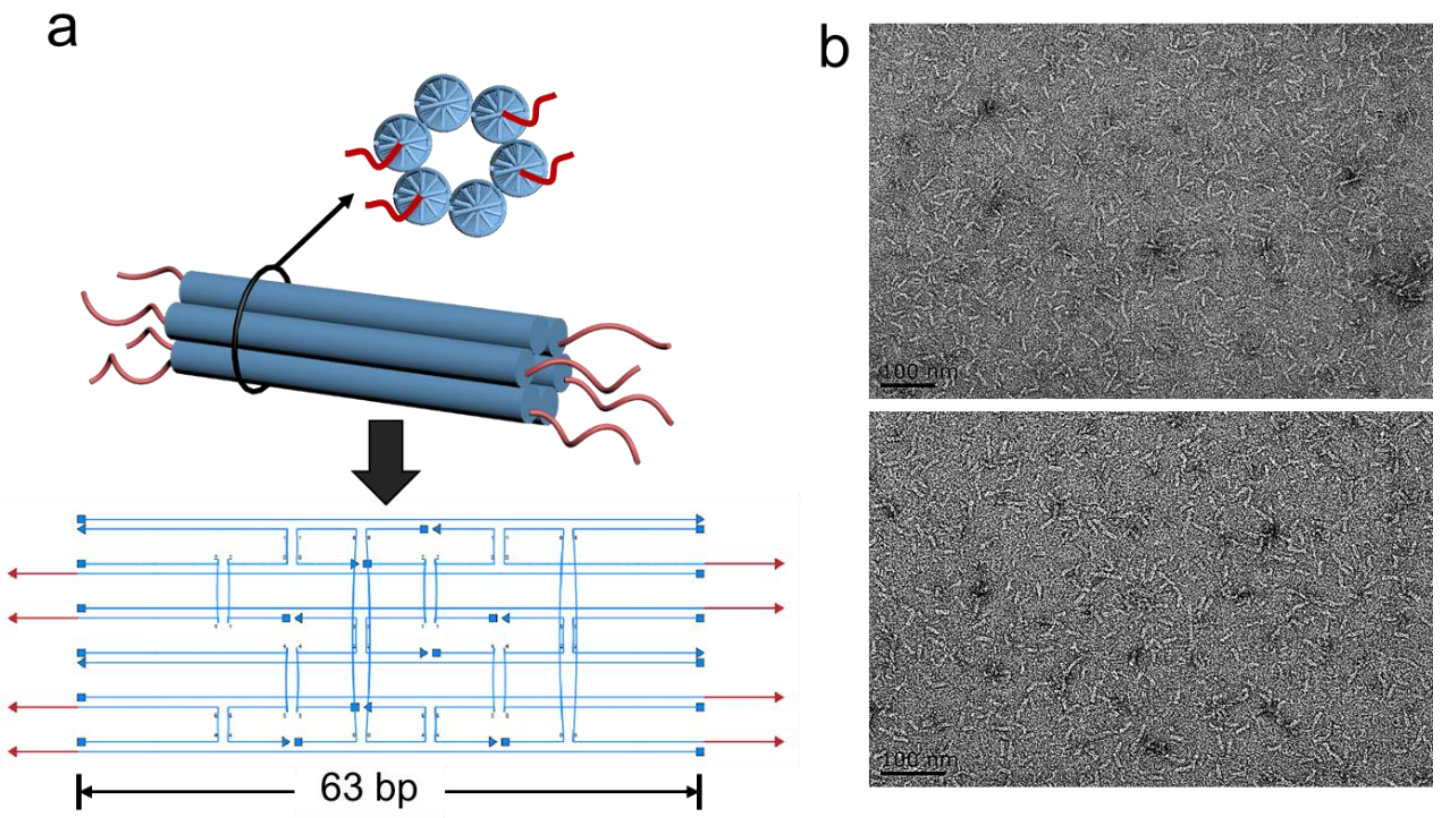

Figure S3. (a) The schematic illustration shows the design of six-helix bundle (6HB). (b) Representative TEM images for the $6 \mathrm{HB}-\mathrm{R}_{63}$. 


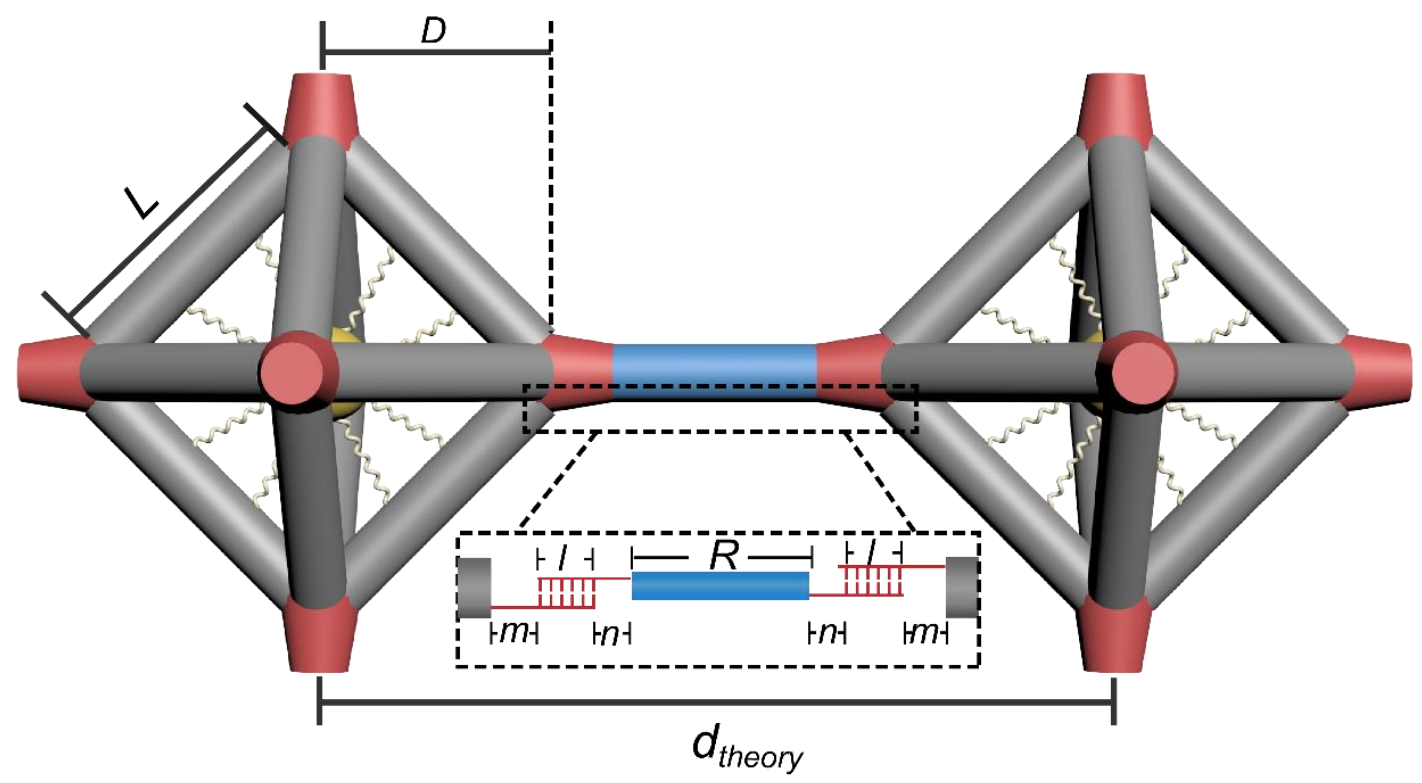

Figure S4. Geometric model for the octahedral DOFs and the binding details of the two octahedra nearby. The octahedral DOFs composed of twelve 6HB bundles with length $L=28.56 \mathrm{~nm}$. The half wide of the octahedra is set to $D=22.19 \mathrm{~nm}$ taking the chamfering of the vertex into account. The binding region of the two octahedra nearby was divided into seven parts, including backbone of DNA rods (denoted by “ $R$ ”), two recognition parts (denoted by “" $l$ "), two flexible parts of DOFs' sticky ends (denoted by " $m$ ") and two of DNA rods'(denoted by “ $n$ "). $d_{\text {theory }}$ is the theory interparticle distance by theoretical calculation. 

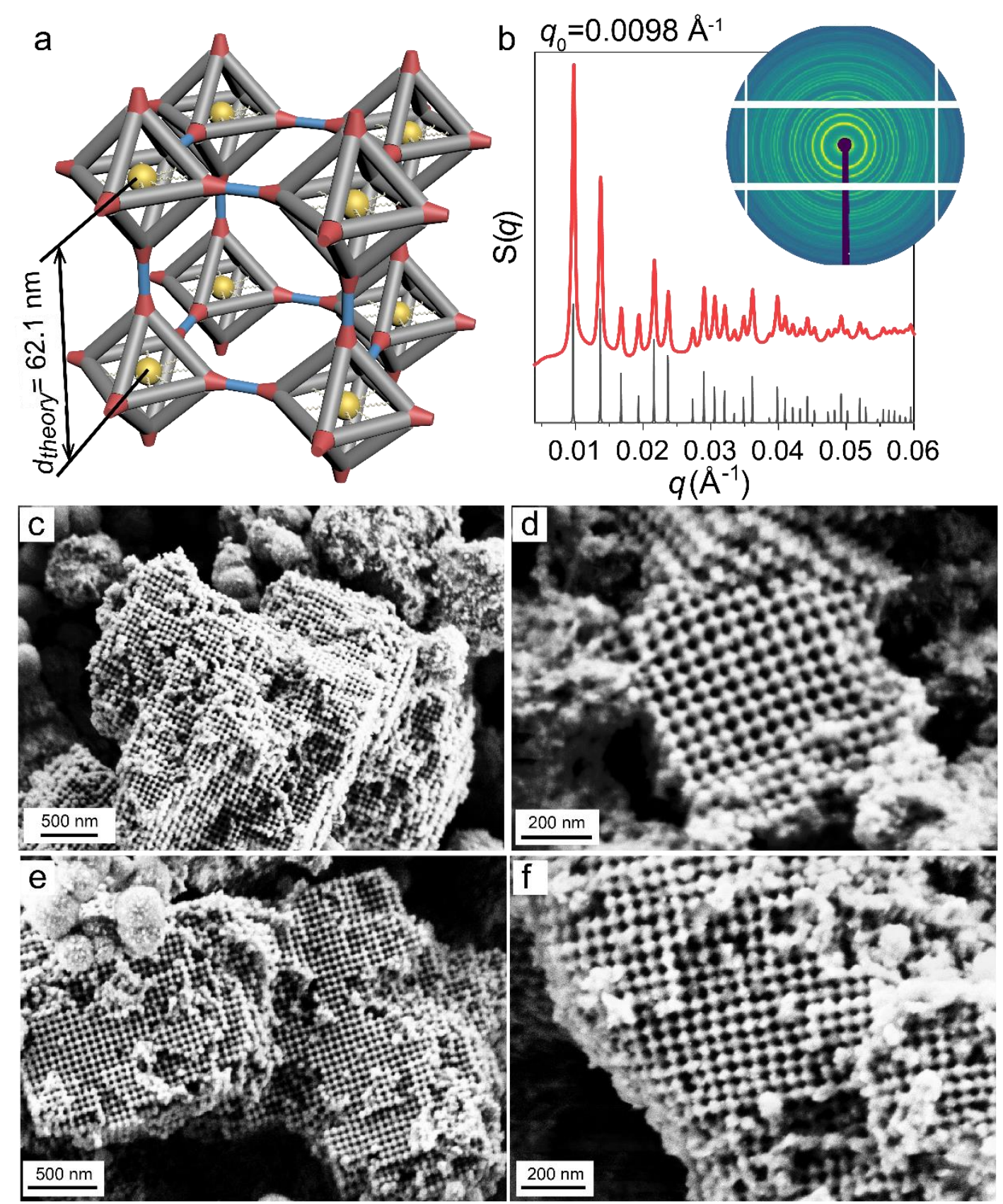

Figure S5. (a) Model of DOF-4DH-R 36 with $d_{\text {theory }}=62.1 \mathrm{~nm}$. (b) The plots of structure factors $\mathrm{S}(q)$ versus $q$ for DOF-4DH-R 36 . Top right panels showed the $2 \mathrm{D}$ rings from SAXS measurement. (c-f) Representative SEM images of silica-infused crystals (DOF4DH-R 36 ). 

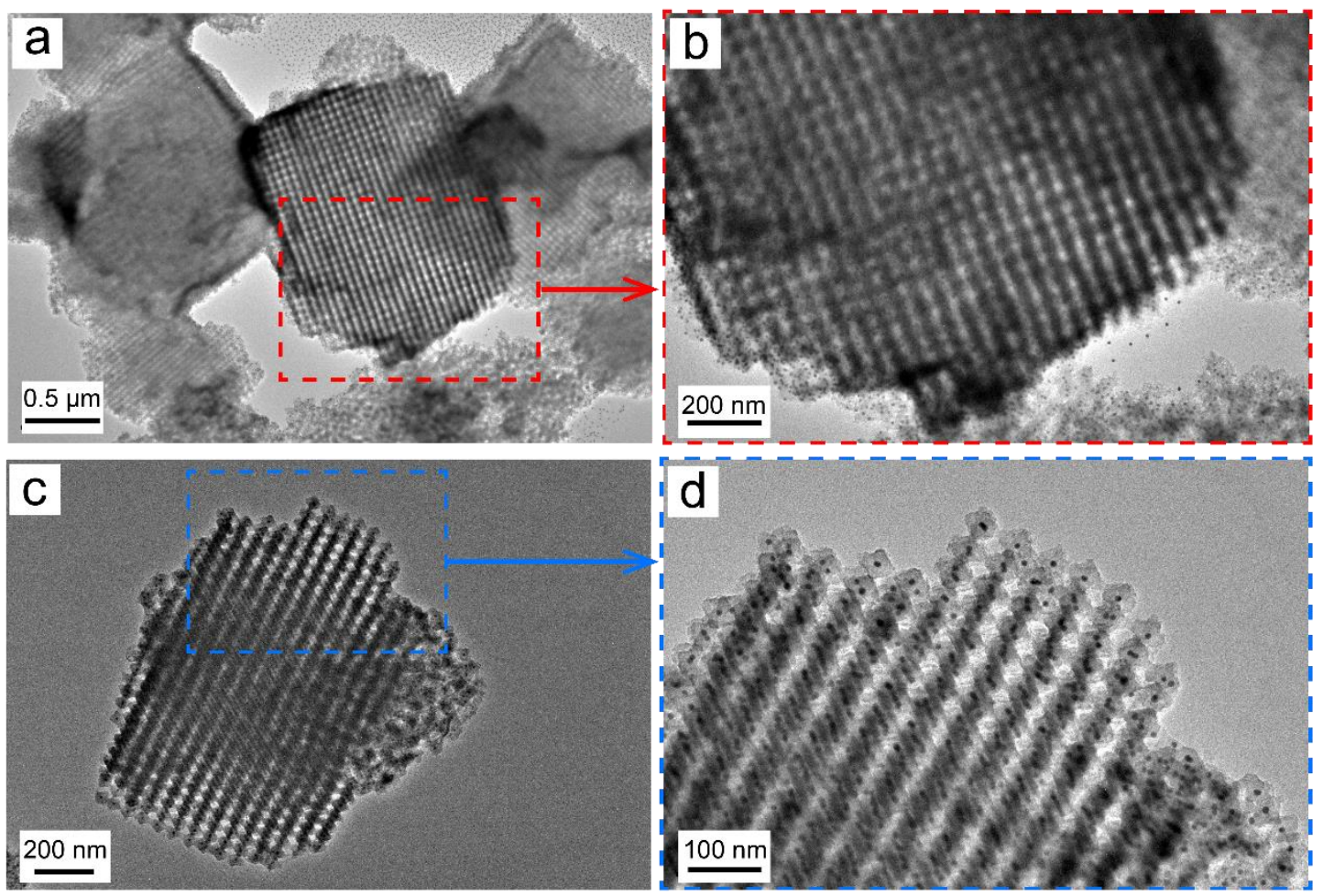

Figure S6. Representative TEM images of silica-infused crystals (DOF-4DH-R36). 

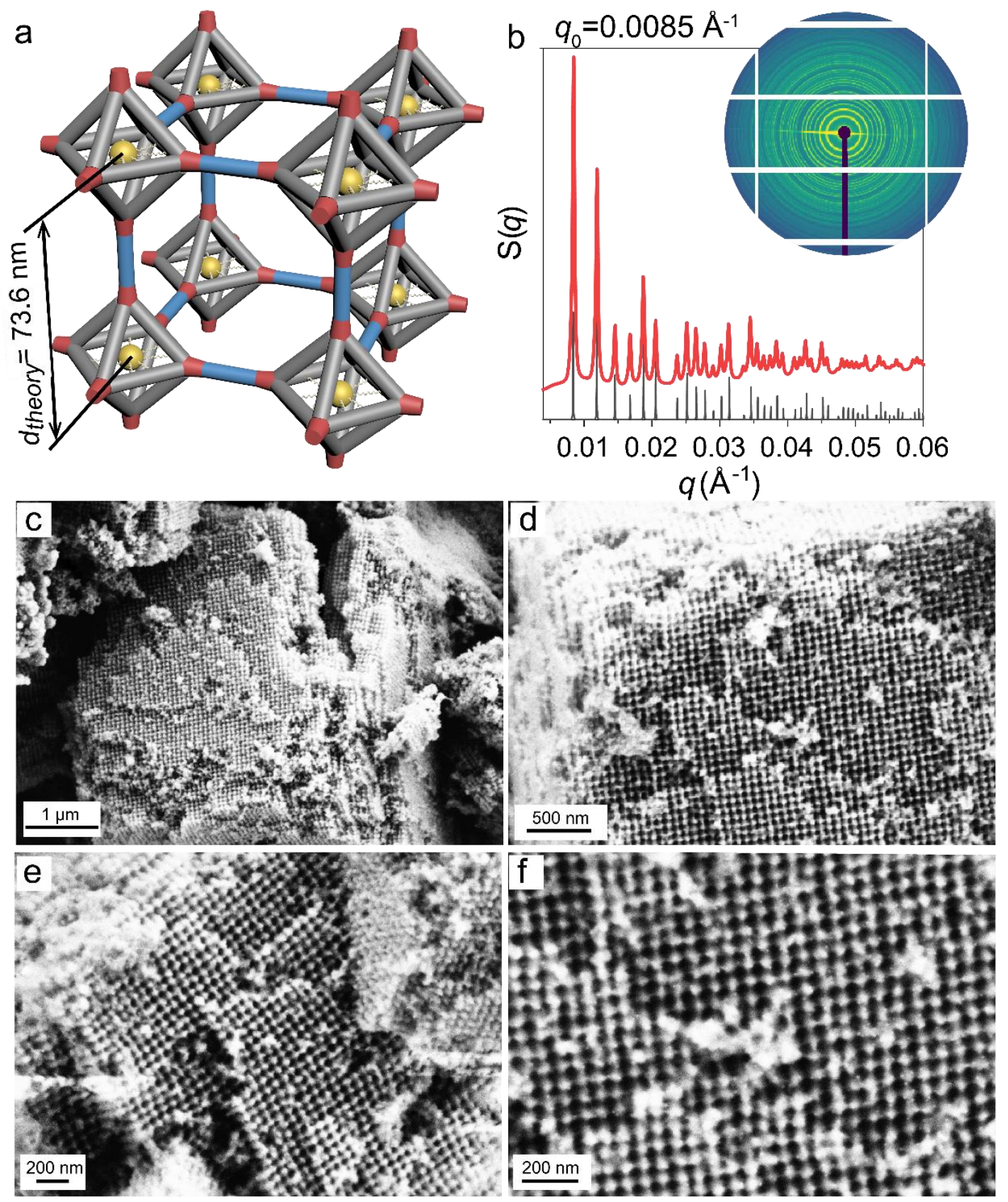

Figure S7. (a) Model of DOF-6HB-R 63 with $d_{\text {theory }}=73.6 \mathrm{~nm}$. (b) The plots of structure factors $\mathrm{S}(q)$ versus $q$ for DOF-6HB- $\mathrm{R}_{63}$. Top right panels showed the $2 \mathrm{D}$ rings from SAXS measurement. (c-f) Representative SEM images of silica-infused crystals (DOF6HB-R 63$)$. 

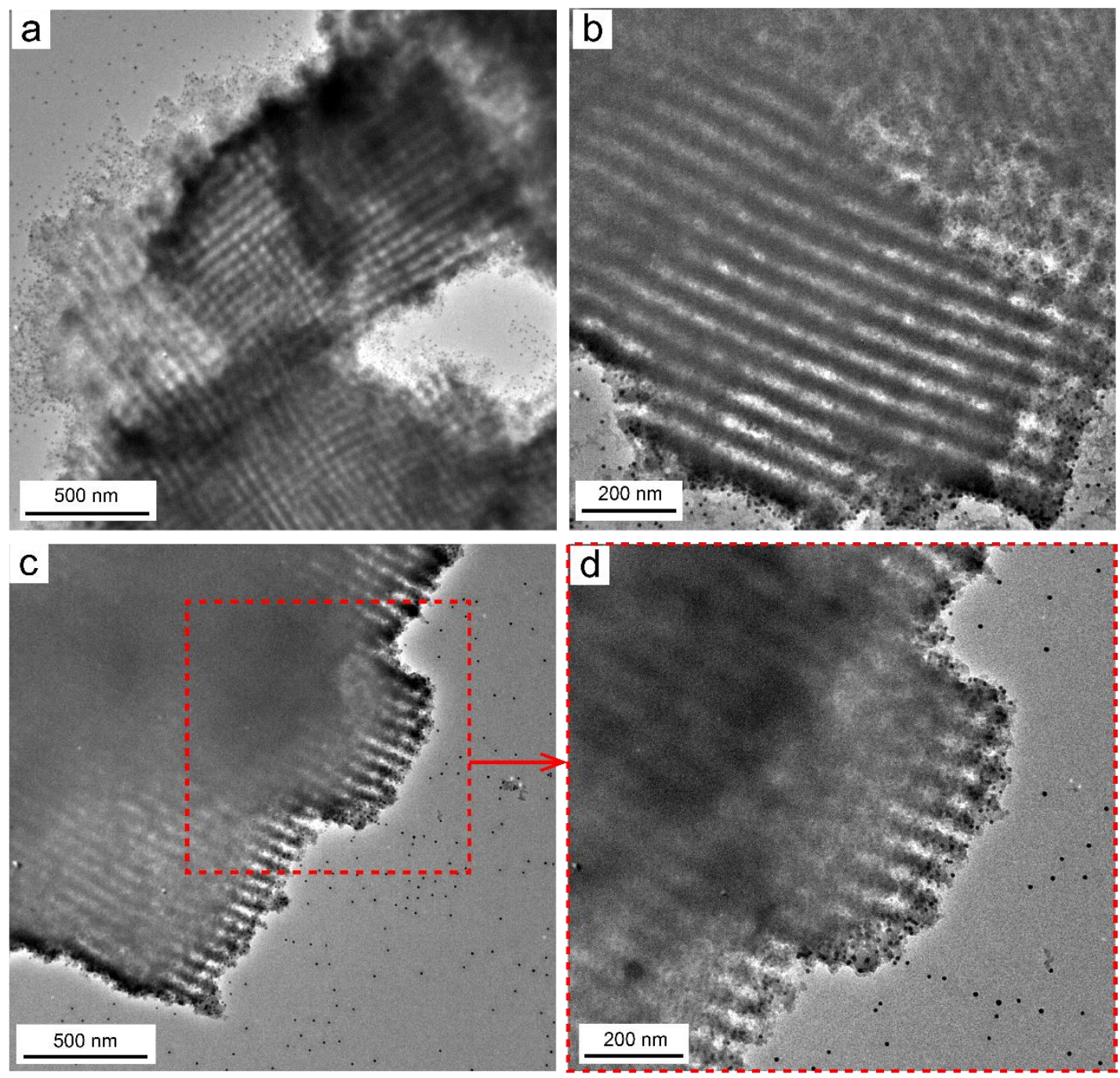

Figure S8. Representative TEM images of silica-infused crystals (DOF-6HB-R63). 


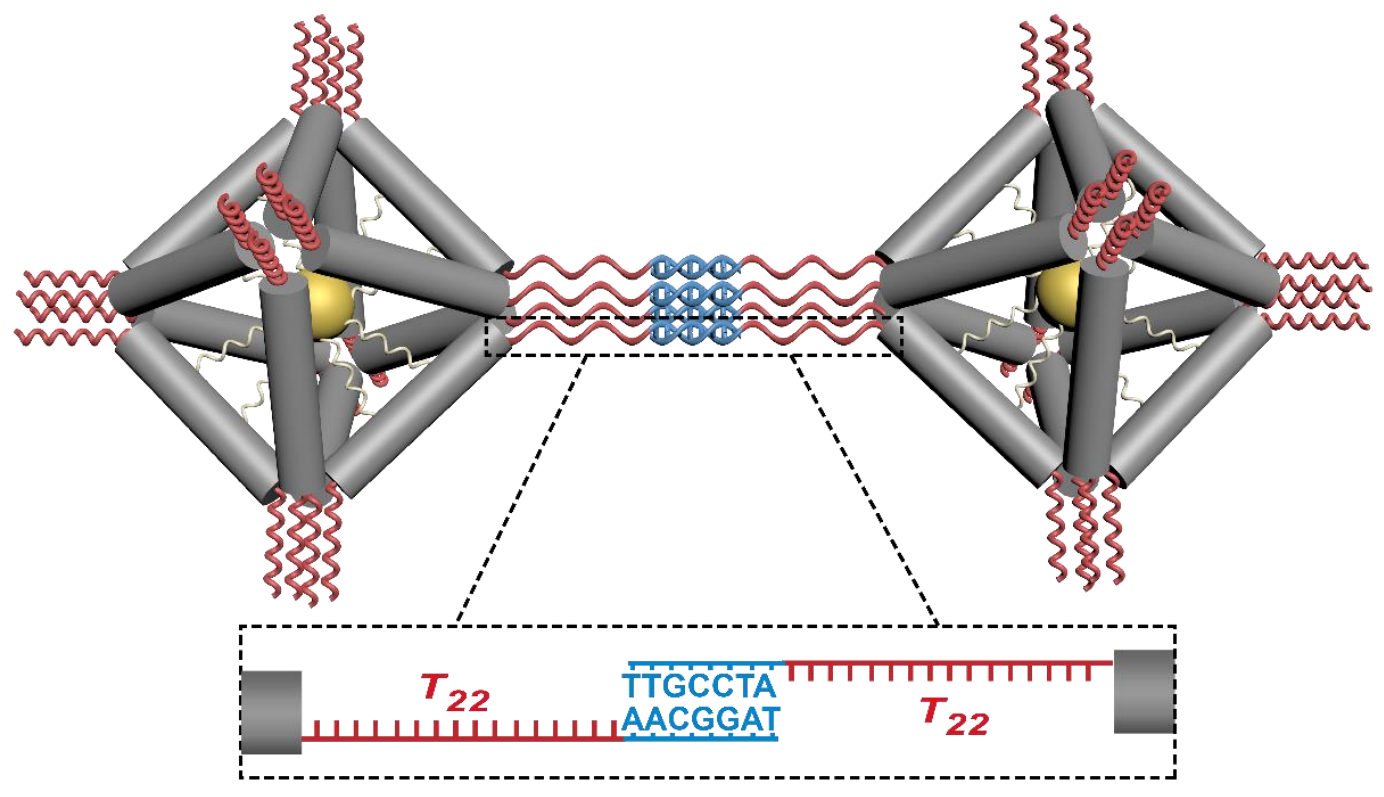

Figure S9. Schematic illustration shows the binding details of the two octahedra nearby of reported DOF direct assembly. ${ }^{6}$ 

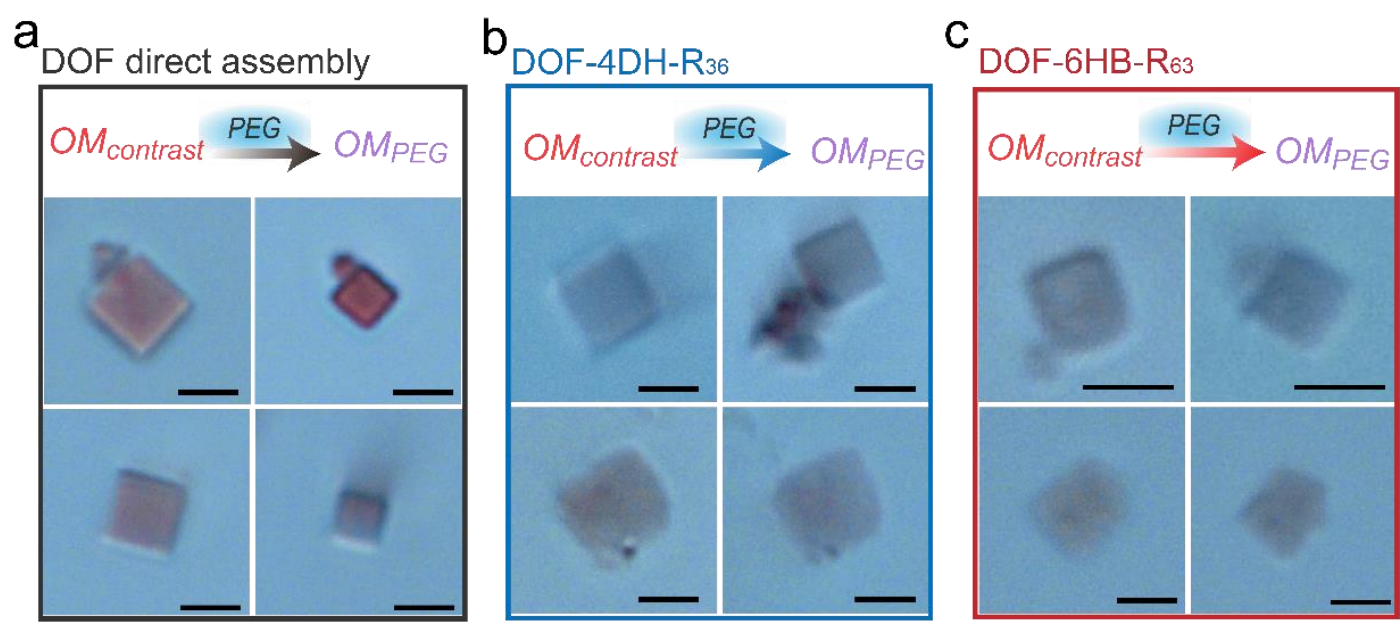

Figure S10. Optical microscopy (OM) images of (a) DOF direct assembly, (b) DOF$4 \mathrm{DH}-\mathrm{R}_{36},(\mathrm{c})$ DOF-6HB-R 63 before (left, $O_{\text {contrast }}$ ) and after (right, $O M_{P E G}$ ) the stimuli. $2 \mu \mathrm{L}$ prepared nanoparticles/DNA lattices was dropped on a glass slide, then $3 \mu \mathrm{L} 30 \%$ w/v PEG (M.W. $=2000$, contains $0.675 \mathrm{M} \mathrm{NaCl}, 10 \mathrm{mM}$ phosphate) was added to adjust the environment to $\sim 18 \% \mathrm{w} / \mathrm{v}$ PEG (contains $\sim 0.3 \mathrm{M} \mathrm{NaCl}, \sim 5.0 \mathrm{mM}$ magnesium acetate). The response of a particular crystal before and after the stimuli was observed by OM. To avoid the influence of large amounts of liquid evaporation, the sample status was recorded within 4 5 min. The DOF direct assembly appears to contract significantly after the stimuli, while this change is not evident in DOF-DNA rod systems. Scale bars, $5 \mu \mathrm{m}$. 

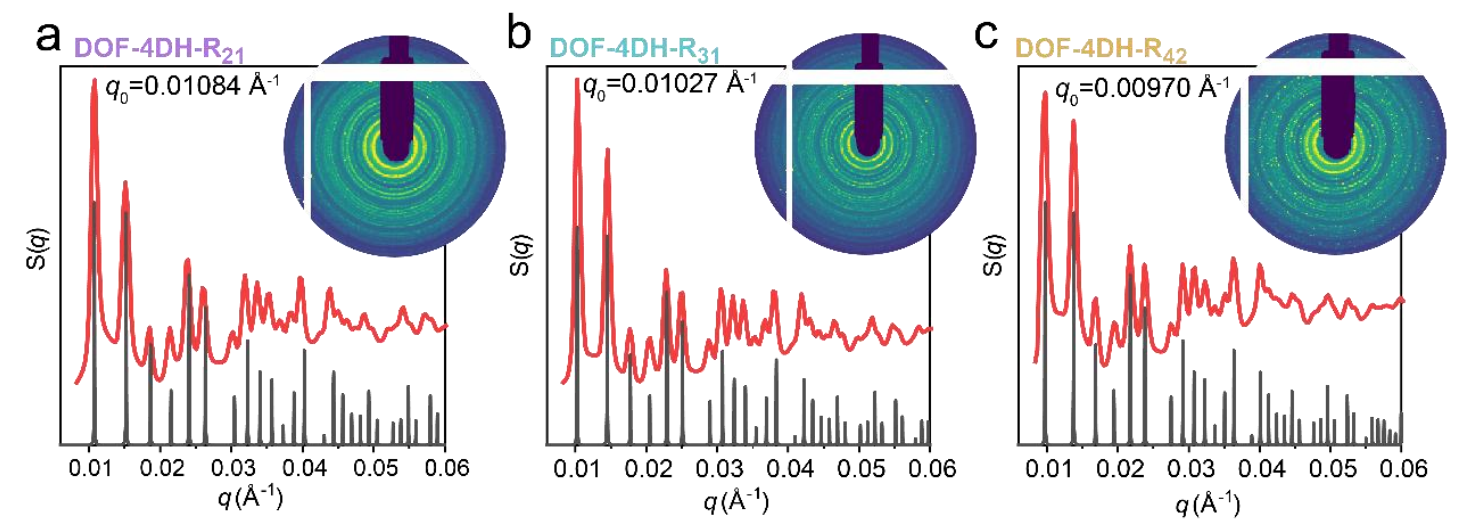

Figure S11. The plots of structure factors $\mathrm{S}(q)$ versus $q$ for lattices formed by octahedral DOFs mediated by (a) 4DH-R 21 ; (b) 4DH-R 31 ; (c) 4DH-R 42 . Top right panels showed the $2 \mathrm{D}$ rings from SAXS measurement. 


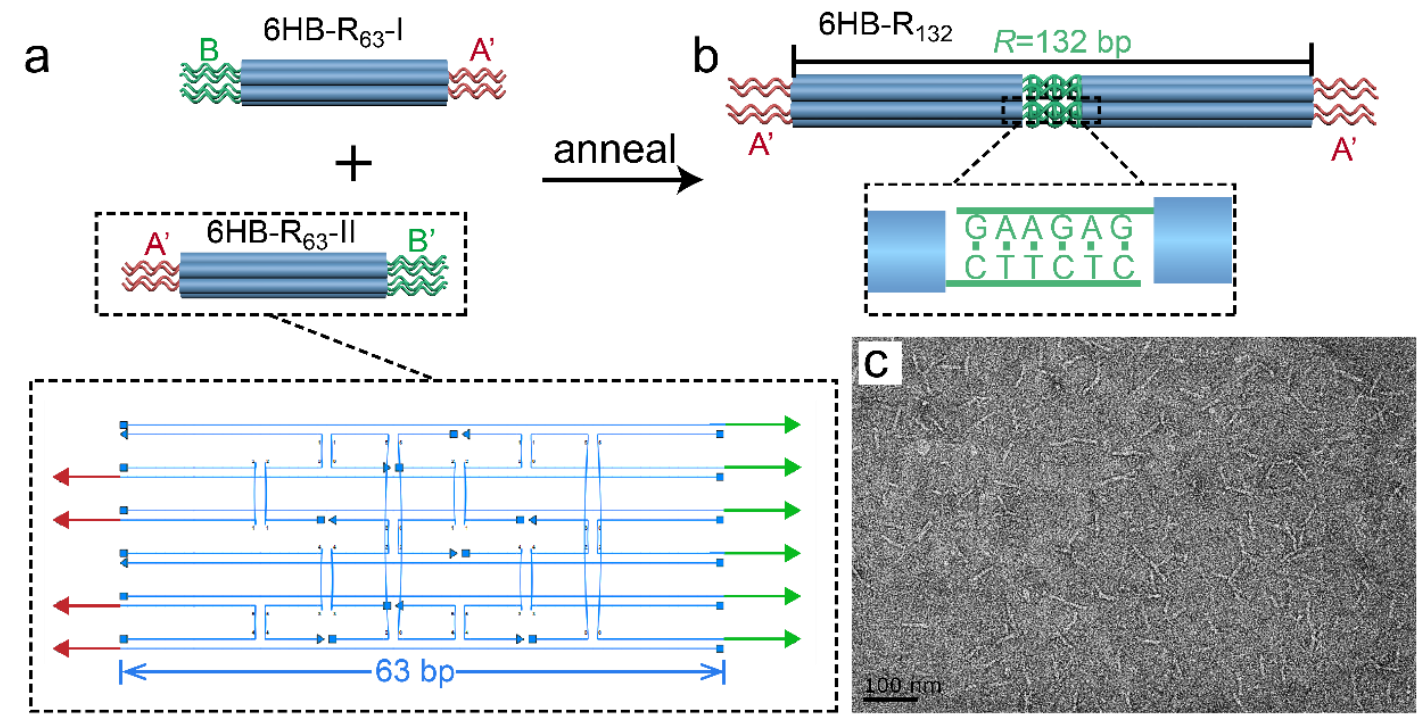

Figure S12. Schematic illustration for forming $6 \mathrm{HB}-\mathrm{R}_{132}$ with $6 \mathrm{HB}-\mathrm{R}_{63}$. (a)

Schematic of two kinds of $6 \mathrm{HB}-\mathrm{R}_{63}\left(6 \mathrm{HB}-\mathrm{R}_{63}-\mathrm{I}\right.$ and $\left.6 \mathrm{HB}-\mathrm{R}_{63}-\mathrm{II}\right)$ with complementary sticky ends for further assembly (green line denoted by "B" and " $\mathrm{B}$ ",). The bottom part showed the design detail of $6 \mathrm{HB}-\mathrm{R}_{63}$ monomer. (b) Schematic illustration of the formed $6 \mathrm{HB}-\mathrm{R}_{132}$. Zoomed in image showed the connection detail and DNA sequences. (c) Representative TEM images of the $6 \mathrm{HB}-\mathrm{R}_{132}$. 

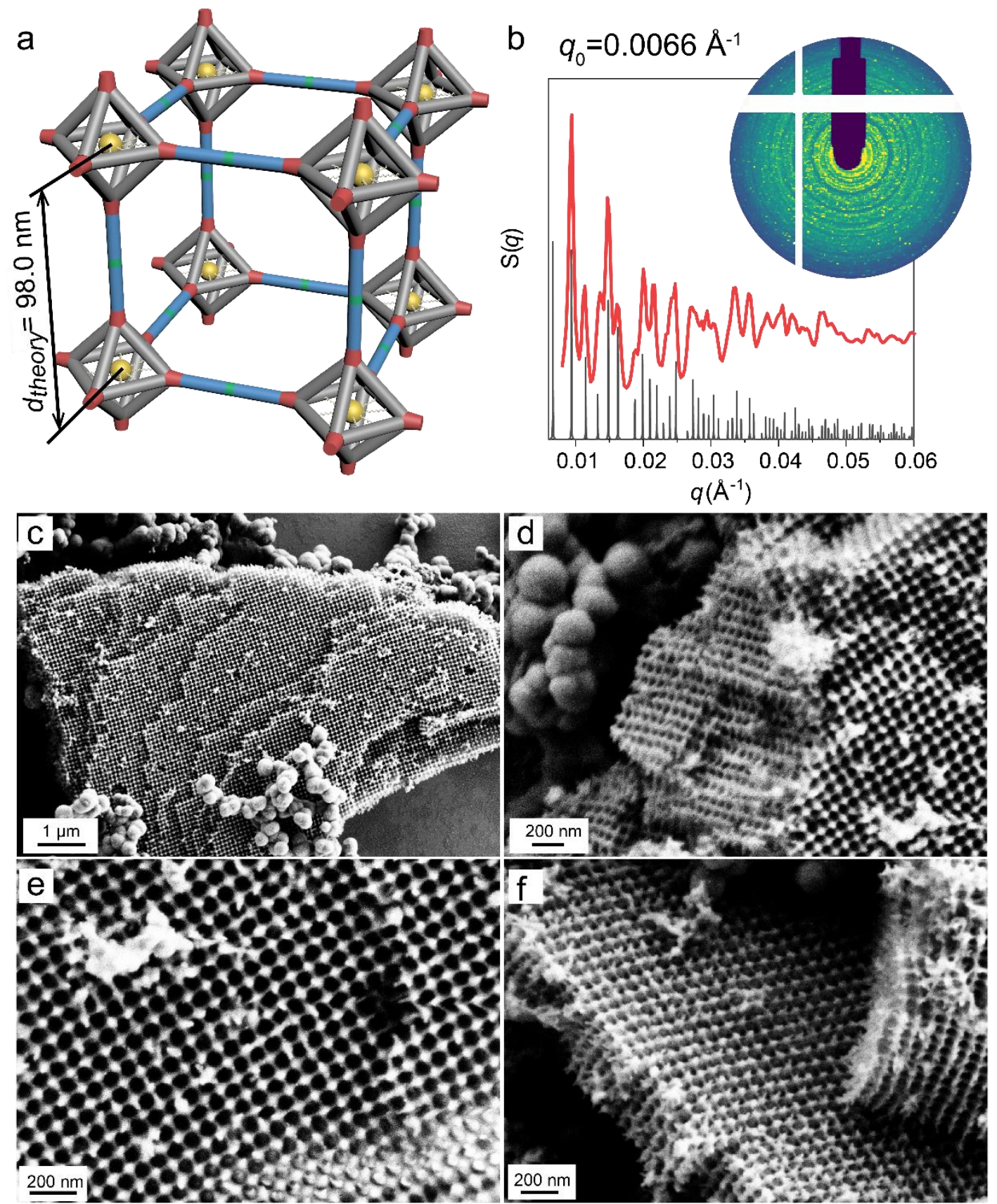

Figure S13. (a) Model of DOF-6HB-R ${ }_{132}$ with $d_{\text {theory }}=98.0 \mathrm{~nm}$. (b) The plots of structure factors $\mathrm{S}(q)$ versus $q$ for DOF-6HB- $\mathrm{R}_{132}$. Top right panels showed the 2D rings from SAXS measurement. (c-f) Representative SEM images of silica-infused crystals (DOF-6HB-R 132 ). 

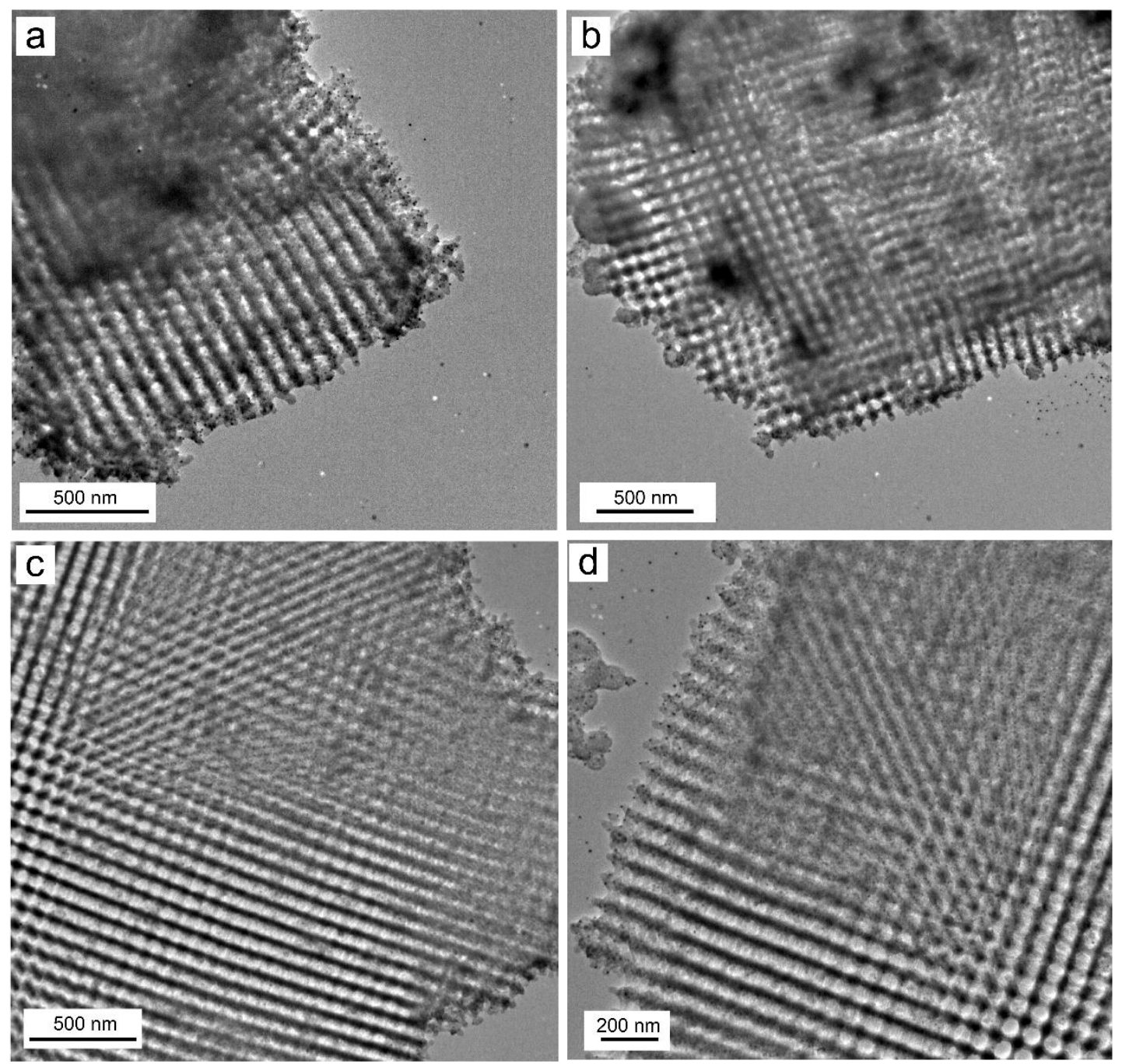

Figure S14. Representative TEM images of silica-infused crystals (DOF-6HBR132). 
IX. Supplementary Tables

\begin{tabular}{|c|c|c|}
\hline \multicolumn{2}{|c|}{ Name } & Base number \\
\hline \multicolumn{2}{|c|}{$4 \mathrm{DH}-\mathrm{R}_{36}$} & 84 \\
\hline \multirow{2}{*}{$4 \mathrm{DH}-\mathrm{R}_{36}$-SsDNA } & $\mathrm{I}$ & 42 \\
\cline { 2 - 3 } & $\mathrm{I}$ & 42 \\
\hline $4 \mathrm{DH}-\mathrm{R}_{21}$ & 58 \\
\hline $4 \mathrm{DH}-\mathrm{R}_{31}$ & 78 \\
\hline $4 \mathrm{DH}-\mathrm{R}_{42}$ & 100 \\
\hline
\end{tabular}

Table S1. The details of the duplex DNA of 4DH analyzed in the PAGE gel. 


\begin{tabular}{|c|c|c|c|c|c|}
\hline & \multirow{2}{*}{$\begin{array}{c}\text { Octahedra } \\
m \text { (nt) }\end{array}$} & \multicolumn{2}{|c|}{ DNA rods } & \multirow{2}{*}{$\begin{array}{c}I \\
\text { (bp) }\end{array}$} & \multirow{2}{*}{$\begin{array}{l}d_{\text {theory }} \\
(\mathrm{nm})\end{array}$} \\
\hline & & $R(\mathrm{bp})$ & $n$ (nt) & & \\
\hline DOF-4DH-R ${ }_{36}$ & 0 & 36 & 0 & 8 & 62.1 \\
\hline DOF-6HB-R 63 & 1 & 63 & 1 & 8 & 73.6 \\
\hline DOF-4DH-R ${ }_{21}$ & 0 & 21 & 0 & 8 & 57.0 \\
\hline DOF-4DH-R ${ }_{31}$ & 0 & 31 & 0 & 8 & 60.4 \\
\hline DOF-4DH-R ${ }_{42}$ & 0 & 42 & 0 & 8 & 64.1 \\
\hline DOF-6HB-R ${ }_{132}$ & 3 & 132 & 1 & 8 & 98.0 \\
\hline
\end{tabular}

Table S2. The various parameters of all DOF-DNA rod assemblies studied here. 


\section{Supplementary References}

(1) Auyeung, E.; Macfarlane, R. J.; Choi, C. H.; Cutler, J. I.; Mirkin, C. A. Transitioning DNAengineered nanoparticle superlattices from solution to the solid state.Adv. Mater. 2012, 24, (38), 5181-6. (2) Liu, X.; Zhang, F.; Jing, X.; Pan, M.; Liu, P.; Li, W.; Zhu, B.; Li, J.; Chen, H.; Wang, L.; Lin, J.; Liu, Y.; Zhao, D.; Yan, H.; Fan, C. Complex silica composite nanomaterials templated with DNA origami.Nature 2018, 559, (7715), 593-598.

(3) Nguyen, L.; Doblinger, M.; Liedl, T.; Heuer-Jungemann, A. DNA-Origami-Templated Silica Growth by Sol-Gel Chemistry.Angew. Chem., Int. Ed. 2019, 58, (3), 912-916.

(4) Simmel, S. S.; Nickels, P. C.; Liedl, T. Wireframe and Tensegrity DNA Nanostructures.Acc. Chem. Res. 2014, 47, (6), 1691-1699.

(5) Nickels, P. C.; Wunsch, B.; Holzmeister, P.; Bae, W.; Kneer, L. M.; Grohmann, D.; Tinnefeld, P.; Liedl, T. Molecular force spectroscopy with a DNA origami-based nanoscopic force clamp.Science 2016, 354, (6310), 305-307.

(6) Tian, Y.; Lhermitte, J. R.; Bai, L.; Vo, T.; Xin, H. L.; Li, H.; Li, R.; Fukuto, M.; Yager, K. G.; Kahn, J. S.; Xiong, Y.; Minevich, B.; Kumar, S. K.; Gang, O. Ordered three-dimensional nanomaterials using DNA-prescribed and valence-controlled material voxels.Nat. Mater. 2020, 19, (7), 789-796. 